\title{
From Mutualistic Partner to Hunted Prey?
}

\author{
Erich K. Ritter \\ Department of Mathematics and Statistics, University of West Florida, Pensacola, FL, USA \\ Email: eritter@uwf.edu
}

How to cite this paper: Ritter, E.K. (2019) From Mutualistic Partner to Hunted Prey? Open Journal of Animal Sciences, 9, 88-96. https://doi.org/10.4236/ojas.2019.91008

Received: October 19, 2018

Accepted: January 7, 2019

Published: January 10, 2019

Copyright (C 2019 by author and Scientific Research Publishing Inc. This work is licensed under the Creative Commons Attribution International License (CC BY 4.0).

http://creativecommons.org/licenses/by/4.0/

\begin{abstract}
Sharks and sharksuckers maintain a mutualistic symbiotic relationship; thus, it is surprising to observe a lemon shark, Negaprion brevirostris, killing a sharksucker, Echeneis lucrates, which has been recorded during a dive with lemon sharks. Does this observation indicate that the symbiosis between the two species may shift occasionally? The awkwardness of the recorded kill, combined with its comparatively long duration, suggests this bout be a freak incident, rather than a common occurrence; thus, the mutualistic relationship needs not be questioned. What triggered the bout, however, can only be speculated. Although the caloric value of the killed sharksucker is not known, a feeding-oriented behavior can likely be rejected as the potential cause based on the teleost's rather small size, and an irritation related issue is more likely to have triggered this bout.
\end{abstract}

\section{Keywords}

Irritation, Kill, Mutualistic, Shark, Sharksucker, Symbiosis

\section{Introduction}

Sharksuckers remove parasites from a shark's skin [1] [2] [3], clean wounds and even remove food remnants from between their teeth [4]. In return, sharksuckers benefit from feeding on food particles after a shark's bout, keep mostly attached to a shark, reducing energy expenditure and gaining protection. Although advantages can go both ways in a symbiotic mutually beneficial relationship, sharksuckers can also be very irritating to their clients when sucking on sensitive areas along a shark's body [2] [3] [5]. Such irritation can lead to rather extreme reactions by the shark, such as leaping out of the water and falling back onto the surface with the irritated body area first [6], chafing the irritated body part on the bottom [3], or scooping off sharksuckers with a pectoral fin [7].

Although sharksucker irritation and the concurrent reaction by the shark can be quite prominent, the relationship between the two is considered mutualistic. 
However, the observation presented in this event where a lemon shark, Negaprion brevirostris, kills a sharksucker, Echeneis lucrates, seems to contradict this mutualistic relationship. This act is even more surprising, bearing in mind that smaller sharksuckers are tolerated to such an extent that they can safely remove food particles from between a shark's upper teeth, and are even invited to do so [4]. Such a kill then seems to make the relationship between the two species unclear. Overall, there are a variety of examples, especially within cleaning interactions, where the exact nature of a relationship is also less clear [8]. Thus, the question to be answered is whether the relationship between lemon sharks and sharksuckers could temporarily turn into a somewhat antagonistic relationship should irritation be overwhelming? That mutualistic relationship can temporarily turn into a neutral or antagonistic relationship, which has been shown in the past [9].

The following analysis focuses on the different stages and effectiveness of the kill, and their potential meaning regarding the overall relationship between the two species.

\section{Materials and Methods}

The bout was recorded at a site called "Tiger Beach," in the Bahamas, at a depth of about $12 \mathrm{~m}$, over a sandy bottom. The incident was video recorded by two divers from two different angles during different parts of the bout; thus, the description of the event is based on evidence from both clips. The clips were taken a 1080 High Res at 25 frames per second. The clips were enhanced using Apple Final Cut Pro X software, where necessary, and single frames were further augmented using Adobe Photoshop CS 5, where needed.

The lemon shark's length was estimated to be around $2.5 \mathrm{~m}$, and it was part of a group of about 30 sharks. The sharksucker was about $30 \mathrm{~cm}$ long, similar to previous observations [4]. The incident took place in December, late in the day just before sunset.

The presented bout was the second sharksucker kill by a lemon shark, observed by the author in 10 years and more than 300 diving hours at the same site, where lemon sharks are the predominant shark species year-round.

\section{Results}

The duration of the bout took 41 seconds from the initial contact between the lemon shark and the sharksucker to the last visible gulp motion. During that, the shark lost its hold on the sharksucker several times (Figure 1(a), Figure 1(b)). The released teleost appeared to be immobile when the shark lost its grip approximately 12 seconds into the bout (Figure $1(\mathrm{c})$ ). Until then, the shark shook its head very rapidly while gripping the sharksucker. From that point on, the shark's head movements mostly were reduced to attempts at swallowing its prey (Figure 1(d), Figure 1(f)).

In addition to the sharksucker that was consumed, five others were either 


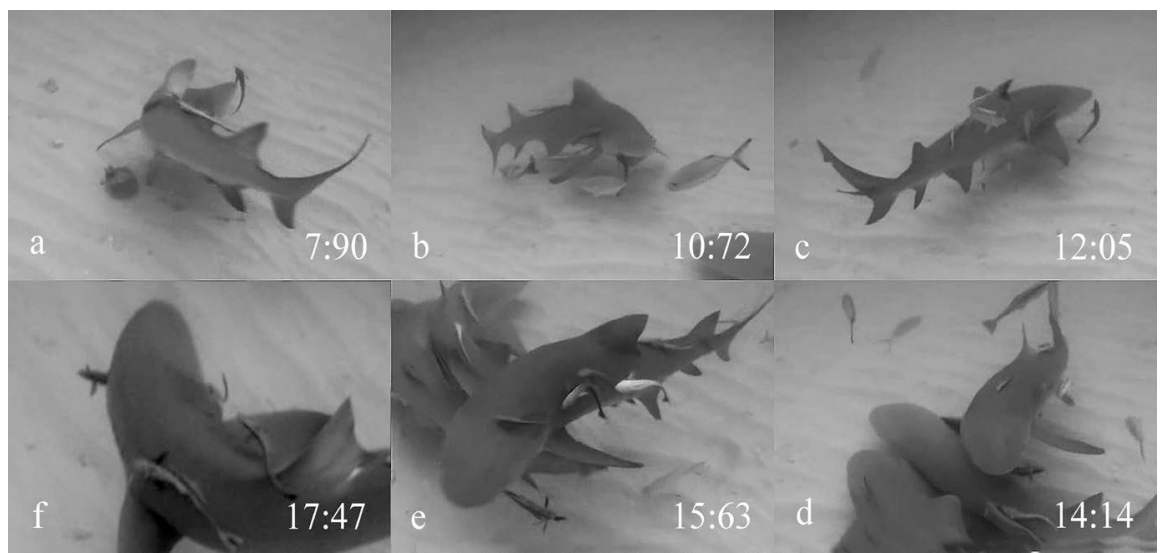

Figure 1. Individual frames in sequence of the lemon shark trying to kill and swallow a sharksucker. (a) The lemon shark tries to grab the sharksucker; (b) The sharksucker is in a likely position to get sucked in; (c) The shark loses its hold on the sharksucker again; (d) Other sharks are not interested in the ongoing bout despite the close contact with them. (e) The shark holds on to the tail of the sharksucker; (f) Ongoing attempt to position and swallow the sharksucker. Numbers reflect duration in fraction of seconds from the beginning of the bout. Frames were taken from the video by T. Conrad.

attached to the lemon shark's body or were swimming in very close proximity (e.g., Figure 1(e)). The sizes of the different sharksuckers were similar to the one that was killed, exceeding the size that would allow them to clean between the lemon shark's upper teeth [4].

During the bout, the shark in question crossed three other lemon sharks of similar size which did not react to the ongoing feeding attempt (Figure 1(d)). Shortly after that, two more lemon sharks were passed of which one showed some interest and started to follow briefly but did not attempt to challenge the feeding shark.

\section{Discussion}

The relationship between sharks and sharksuckers dates back to the Eocene-Oligocene period [10] [11] [12]. From early on it was noted that sharksuckers removed ectoparasites from a shark's skin, clean wounds, and remove food particles from between a lemon shark's upper teeth [4]. Although mouth cleaning ceases once a sharksucker is too large to enter the shark's mouth, removing parasites and cleaning wounds remain within the teleost's behavioral repertoire [4] However, it is not only the shark who benefits from sharksuckers, these teleosts also profit not just from feeding on ectoparasites [13] but also from consuming food particles and pieces after a shark's hunting bout or what has been stirred up [14], and even from sieving through sands released through a shark's gills should it have sucked in food from a sandy bottom (pers. obs.). Since a sharksucker is attached to its client most of the time, it also saves energy that would otherwise be used to swim. Another advantage a sharksucker gains from being attached to a shark lays in its protection from other predators [15]. Although it cannot be excluded that sharksuckers are never picked off from a 
shark's surface, this has not been observed thus far. Therefore, merely being attached to a shark might be protection enough for a sharksucker. Should that not be the case, a sharksucker would have to be grabbed off another shark, and physical contact between two sharks would then be inevitable. Such a scenario would likely trigger a potentially dangerous antagonistic reaction from the hosting shark.

The benefits a shark and a sharksucker gain from each other stand in stark contrast to the idea that a client would kill one of its cleaners when given a chance. This perspective suggests that the observed kill may be a freak incident rather than a regular event, which could explain why some elements of the bout do not seem as fluid as one would expect should killing sharksuckers by lemon sharks be a regular occurrence.

\subsection{Bout Behavior}

Sharks either suck in prey and swallow it whole [16] [17] [18] or gouge and bite a piece out of a prey should it be larger than the shark's bite volume or gape size [19] [20] [21]. Considering the small size of the sharksucker, which is less than the shark's bite volume, the bout qualifies as suction feeding. However, the shark was either inefficient in sucking the prey in, or the teleost's position was not aligned with the shark's longitudinal axis to perform an effective suction motion [22] [23] [24]. This inability could explain the prolonged duration taken to manipulate the prey for swallowing.

Although vigorous head shaking during bouts is typically used by sharks to immobilize their prey [25] [26] [27], the rather small size of the sharksucker makes the observed degree of shaking appear excessive. If we assume that the initial grabbing of the sharksucker did not lead to severe injuries probably due to insufficient teeth piercing, this could have left the sharksucker with the possibility of escape and wiggle its way out from between the shark's jaws. This scenario could explain the sideways head motions of the shark to grab the sharksucker again firmly. Likewise, the water pressure created by the shark's sideways head motion could have also pushed the sharksucker farther away from the shark's reach. The suction mechanism of sharks aims towards the front of their snouts but not the side. So, if the potential prey is positioned to the side of a shark's snout, the animal needs to turn, at least the front of its body, before any strong suction can ensue, as it may have happened in this bout.

\subsection{Weighing the Value of a Mutual Benefit}

From the viewpoint of a beneficial symbiotic relationship, the killing of a member of a mutualistic species cannot easily be understood. It is even more difficult to explain, knowing that younger specimens of the mutualistic sharksuckers are allowed, and sometimes even invited by the shark, to enter its mouth to clean between the upper teeth [4]. To then kill a sharksucker implies that the shark must possess at least some selective feeding inhibition during regular cleaning 
bouts. This reticence may be switched on as soon as sharksuckers trigger the mouth opening of the targeted shark by announcing cleaning intentions through a dance-like behavior [4]. This dance is always performed right in front of a shark's eyes, easily close enough for a shark to grab the sharksucker. However, this does not seem to occur when the sharksucker still has a size that allows cleaning inside the shark's mouth; thus, it appears that at least small sharksuckers are excluded from being preyed on. This raises the question of whether this also is generally the case with larger sharksuckers despite the presented video?

Considering the extensive time spent by the author at this site over the last ten years and only having witnessed one other sharksucker kill by a lemon shark, suggests that feeding on sharksuckers by lemon sharks is indeed not a regular occurrence. Thus, the existence of a mutualistic relationship does not seem to be challenged. However, despite the rarity of this bout, it shows that under certain circumstances even members of a mutualistic species are not saved from being killed and swallowed.

Mutualistic relationships where teleost species act as the cleaners, as with the sharksuckers, in this case, are a common phenomenon [28] [29] [30]. That a client, however, turns on a cleaner and even kills it, is unusual. Should antagonistic behavior occur, it originates more often from the cleaner's side [8] [31] [32]. That lemon sharks are sometimes affected by sharksuckers when they irritate their skin is known [2] [3] [6], but the responses are less drastic than with this recorded kill and only go as far as to remove the sharksucker from the irritated area. It has previously been proposed that predators refrain from hunting cleaners [33], and even if a cleaner has been injured, predators did not seem to go after them [34].

\subsection{Point of No Return}

It took the shark a considerable amount of time to kill and swallow the sharksucker, in comparison with typical hunting and killing activities, which are over within just a few seconds [27]. Although it can be assumed that the described bout is likely a freak incident and not a common occurrence, the motivation behind it should be further explored. It is doubtful that the shark went after the sharksucker for its caloric value. The caloric value of sharksuckers has not yet been measured, but a general value can still be estimated [35] [36] [37]. Putting this amount in perspective with the lemon shark's daily energy intake of between $1.5 \%$ and $2.1 \%$ of its body weight [38] and high metabolism [39] [40] makes the net energy balance of this bout rather low or even negative. This low gain or possible deficit raises the question of why the lemon shark continued its intention to kill the sharksucker and did not cease in the effort once it realized that the sharksucker was difficult to grab. If a rather instinct driven force guided the bout, a continuation of an attempt to capture and kill could be accepted. Should the shark, however, run into problems, as seen for this incident, a termination of the attempt should occur. However, the shark could not have anticipated the problems it would face due to the slenderness of the sharksucker which is chal- 
lenging for a shark with primarily piercing teeth. On the other hand, it could also reflect a self-preservation aspect of not giving potential prey up for conspecifics close by.

The inability of the lemon shark to grab and kill the sharksucker promptly suggests that this bout was indeed a freak incident, and the shark may have been behaving instinctively rather than being in control of its actions; otherwise, the attempt would have likely been terminated. So, what could have caused the presented kill? Were too many sharksuckers attached to the shark, and it intended to reduce the load it was carrying around? Such a scenario seems unlikely. Quite often more sharksuckers are attached to a lemon shark than in this case [4] [5]. The kill does not reflect a typical behavior; otherwise, it would have been observed more frequently than the two times throughout the more than 300 dive hours at the site. This rarity point towards a situation that went beyond common boundaries between the lemon shark and the sharksucker. The simplest scenario would be a situation where irritation of the sensory organs around the lemon shark's head exceeded a tolerable level, while the sharksucker remained too close to the shark's jaws. Could it be that the sharksucker in question tried to clean between the shark's upper teeth and was then accidentally killed? Although such a scenario cannot be excluded, it is somewhat doubtful since the sharksucker was not just beyond the regular size that commonly cleans between a lemon shark's teeth [4] but the shark would then just have swallowed it.

\section{Conclusion}

Despite the lack of similar incidents, the awkwardness of the lemon shark killing the sharksucker suggests the presented bout reflected a rather unusual situation among lemon sharks. The small size of the teleost and the rather long duration it took to swallow the sharksucker finally do not support the idea that the kill was part of the shark's daily needed caloric intake but was likely caused by the teleost irritating the lemon shark.

\section{Acknowledgements}

I thank Thomas Conrad and Kay Knoll for allowing to use their videos to describe this bout.

\section{Conflicts of Interest}

The author declares no conflicts of interest regarding the publication of this paper.

\section{References}

[1] Cressey, R.F. and Lachner, E.A. (1970) The Parasitic Copepod Diet and Life History of Diskfishes (Echeneidae). Copeia, 1970, 310-318. https://doi.org/10.2307/1441652

[2] Ritter, E. (2002) Analysis of Sharksucker, Echeneis naucrates, Induced Behavior Patterns in the Blacktip Shark, Carcharhinus limbatus. Environmental Biology of Fishes, 64, 111-115. https://doi.org/10.1023/A:1019642221755 
[3] Ritter, E. (2011) Use of Sand Ripples to Enhance Chafing in Caribbean Reef Sharks (Carcharhinus perezi) and Blacktip Sharks (Carcharhinus limbatus). Bulletin of Marine Science, 87, 413-419. https://doi.org/10.5343/bms.2010.1082

[4] Ritter, E.K. and Amin, R. (2016) Mouth Cleaning of Lemon Sharks, Negaprion brevirostris, by Sharksuckers, Echeneis naucrates. Copeia, 104, 728-733.

https://doi.org/10.1643/CE-16-431

[5] Amin, R., Ritter, E., Kulldorf, M., Barbas, A. and Schwarzmeier, M. (2016) Sharksuckers, Echeneis naucrates, are Non-Randomly Attached to the Bodies of Lemon Sharks, Negaprion brevirostris: A Spatial Study. Environmental Sciences, 4, 79-93. https://doi.org/10.12988/es.2016.672

[6] Ritter, E.K. and Brunnschweiler, J.M. (2003) Do Sharksuckers, Echeneis naucrates, Induce Jump Behaviour in Blacktip Sharks, Carcharhinus limbatus? Marine and Freshwater Behaviour and Physiology, 36, 111-113. https://doi.org/10.1080/1023624031000119584

[7] Ritter, E.K. (2012) A Rare Use of a Shark's Pectoral Fin? Scooping off a Sharksucker from the Flank. Open Fish Science Journal, 5, 57-59. https://doi.org/10.2174/1874401X01205010057

[8] Cheney, K.L. and Côté, I.M. (2005) Mutualism or Parasitism? The Variable Outcome of Cleaning Symbioses. Biology Letters, 1, 162-165. https://doi.org/10.1098/rsbl.2004.0288

[9] Thompson, J.N. and Cunningham, B.M. (2002) Geographic Structure and Dynamics of Coevolutionary Selection. Nature, 417, 735-738.

https://doi.org/10.1038/nature00810

[10] Micklich, N. (1998) New Information of the Fishfauna of the Frauenweiler Fossil Site. Italian Journal of Zoology, 65, 169-184.

https://doi.org/10.1080/11250009809386809

[11] O’Toole, B. (2002) Phylogeny of the Species of the Superfamily Echeneoidea (Perciformes: Carangoidei: Echeneidae, Rachycentridae, and Coryphaenidae), with an Interpretation of Echeneid Hitchhiking Behaviour. Canadian Journal of Zoology, 80, 596-623. https://doi.org/10.1139/z02-031

[12] Friedman, M., Johanson, Z., Harrington, R.C., Near, T.J. and Graham, M.R. (2013) An Early Fossil Remora (Echeneoidea) Reveals the Evolutionary Assembly of the Adhesion Disc. Proceedings of the Royal Society B, 280, Article ID: 20131200. https://doi.org/10.1098/rspb.2013.1200

[13] Sazima, I., Moura, R.L. and Rodrigues, M.C.M. (1999) A Juvenile Sharksucker, Echeneis naucrates (Echeneidae), Acting as a Station-Based Cleaner Fish. Cybium, 23, 377-380.

[14] Sazima, I. and Grossman, A. (2006) Turtle Riders: Remoras on Marine Turtles in Southwest Atlantic. Neotropical Ichthyology, 4, 123-126. https://doi.org/10.1590/S1679-62252006000100014

[15] Fertl, D. and Landry, A.M. (2009) Remoras. In: Perrin, W.F., Wursig, B. and Thewissen, J.G.M., Eds., Encyclopedia of Marine Mammals, Elsevier, Amsterdam, 942-943. https://doi.org/10.1016/B978-0-12-373553-9.00216-9

[16] Motta, P.J. (2004) Prey Capture Behavior and Feeding Mechanics of Elasmobranchs. In: Carrier, J.C., Musick, J.A. and Heithaus, M.R., Eds., Biology of Sharks and Their Relatives, CRC Press, Boca Raton, 165-202. https://doi.org/10.1201/9780203491317.ch6

[17] Matott, M.P., Motta, P.J. and Hueter, R.E. (2005) Modulation in Feeding Kinematics and Motor Pattern of the Nurse Shark Ginglymostoma cirratum. Environmental 
Biology of Fishes, 74, 163-174. https://doi.org/10.1007/s10641-005-7435-3

[18] Motta, P.J., Hueter, R.E., Tricas, T.C. and Summers, A.P. (2002) Kinematic Analysis of Suction Feeding in the Nurse Shark, Ginglymostoma cirratum (Orectolobiformes, Ginglymostomatidae). Copeia, 2002, 24-38. https://doi.org/10.1643/0045-8511(2002)002[0024:KAOSFI]2.0.CO;2

[19] Long, D.J. and Jones, R.E. (1996) White Shark Predation and Scavenging on Cetaceans in the Eastern North Pacific Ocean. In: Klimley, P.A. and Ainley, D., Eds., Great White Sharks: The Biology of Carcharodon carcharias. Academic Press, Cambridge, 293-307. https://doi.org/10.1016/B978-012415031-7/50028-8

[20] Ritter, E.K. and Levine, M. (2005) Bite Motivation of Sharks Reflected by the Wound Structure on Humans. American Journal of Forensic Medicine and Pathology, 26, 136-140.

[21] Heupel, M.R., Knip, D.M., Simpfendorfer, C.A. and Dulvy, N.K. (2014) Sizing up the Ecological Role of Sharks as Predators. Marine Ecology Progress Series, 495, 291-298. https://doi.org/10.3354/meps10597

[22] Lowry, D. and Motta, P.J. (2008) Relative Importance of Growth and Behaviour to Elasmobranch Suction-Feeding Performance over Early Ontogeny. Journal of the Royal Society Interface, 5, 641-652. https://doi.org/10.1098/rsif.2007.1189

[23] Wilga, C.D. and Sanford, C.P. (2008) Suction Generation in White-Spotted Bamboo Sharks Chiloscyllium plagiosum. Journal of Experimental Biology, 211, 3128-3138. https://doi.org/10.1242/jeb.018002

[24] Ramsay, J.B. and Wilga, C.D. (2017) Function of the Hypobranchial Muscles and Hyoidiomandibular Ligament during Suction Capture and Bite Processing in White-Spotted Bamboo Sharks, Chiloscyllium plagiosum. Journal of Experimental Biology, jeb-165290.

[25] Wilga, C.D. and Motta, P.J. (1998) Feeding Mechanism of the Atlantic Guitarfish Rhinobatos lentiginosus. Modulation of Kinematic and Motor Activity. Journal of Experimental Biology, 201, 3167-3183.

[26] Whitenack, L.B., Simkins, D.C. and Motta, P.J. (2001) Biology Meets Engineering: The Structural Mechanics of Fossil and Extant Shark Teeth. Journal of Morphology, 272, 169-179. https://doi.org/10.1002/jmor.10903

[27] Ritter, E. and Munoz, A. (2018) How to Kill Another Shark: A First Inside Look at a Potential Hunting Strategy of a Galapagos Shark. Open Fish Science Journal, 11, 46-51. https://doi.org/10.2174/1874401X01811010046

[28] Bshary, R. (2003) The Cleaner Wrasse, Labroides dimidiatus, Is a Key Organism for Reef Fish Diversity at Ras Mohammed National Park, Egypt. Journal of Animal Ecology, 72, 169-176. https://doi.org/10.1046/j.1365-2656.2003.00683.x

[29] Grutter, A.S., Murphy, J.M. and Choat, J.H. (2003) Cleaner Fish Drives Local Fish Diversity on Coral Reefs. Current Biology, 13, 64-67. https://doi.org/10.1016/S0960-9822(02)01393-3

[30] Clague, G.E., Cheney, K.L., Goldizen, A.W., McCormick, M.I., Waldie, P.A. and Grutter, A.S. (2011) Long-Term Cleaner Fish Presence Affects Growth of a Coral Reef Fish. Biology Letters, 7, 863-865. https://doi.org/10.1098/rsbl.2011.0458

[31] Bansemer, C., Grutter, A.S. and Poulin, R. (2002) Geographic Variation in the Behaviour of the Cleaner Fish Labroides dimidiatus (Labridae). Ethology, 108, 353-366. https://doi.org/10.1046/j.1439-0310.2002.00777.x

[32] Bshary, R. and Schäffer, D. (2002) Choosy Reef Fish Select Cleaner Fish that Provide High-Quality Service. Animal Behaviour, 63, 557-564.

https://doi.org/10.1006/anbe.2001.1923 
[33] Trivers, R.L. (1971) The Evolution of Reciprocal Altruism. Quarterly Review of Biology, 46, 35-57. https://doi.org/10.1086/406755

[34] Losey, G.S. (1987) Cleaning Symbiosis. Symbiosis, 4, 229-258.

[35] Hartman, K.J. and Brandt, S.B. (1995) Estimating Energy Density of Fish. Transactions of the American Fisheries Society, 124, 347-355. https://doi.org/10.1577/1548-8659(1995)124<0347:EEDOF>2.3.CO;2

[36] Bethea, D.M., Hale, L., Carlson, J.K., Cortés, E., Manire, C.A. and Gelsleichter, J. (2007) Geographic and Ontogenetic Variation in the Diet and Daily Ration of the Bonnethead Shark, Sphyrna tiburo, from the Eastern Gulf of Mexico. Marine Biology, 152, 1009-1020. https://doi.org/10.1007/s00227-007-0728-7

[37] Wuenschel, M.J., Jugovich, A.R. and Hare, J.A. (2006) Estimating the Energy Density of Fish: The Importance of Ontogeny. Transactions of the American Fisheries Society, 135, 379-385. https://doi.org/10.1577/T04-233.1

[38] Cortés, E. and Gruber, S.H. (1990) Diet, Feeding Habits and Estimates of Daily Ration of Young Lemon Sharks, Negaprion brevirostris (Poey). Copeia, 1990, 204-218. https://doi.org/10.2307/1445836

[39] Nixon, A.J. and Gruber, S.H. (1988) Diel Metabolic and Activity Patterns of the Lemon Shark (Negaprion brevirostris). Journal of Experimental Zoology, 248, 1-6. https://doi.org/10.1002/jez.1402480102

[40] Bushnell, P.G., Lutz, P.L. and Gruber, S.H. (1989) The Metabolic Rate of an Active, Tropical Elasmobranch, the Lemon Shark (Negaprion brevirostris). Experimental Biology, 48, 279-283. 